\title{
COMPLEX MOTIONS IN PLANETARY NEBULAE
}

\author{
VINCENT ICKE \\ Sterrewacht Leiden, Postbus 9513, 2300 RA Leiden, The Netherlands
}

\section{The Hydrodynamic Model}

I have made an extensive series of numerical simulations of aspherical PNs. This interactingwinds model consists of a point source of fast tenuous gas embedded in a flattened cloud of dense slow gas which is two-dimensional and cylindrically symmetric. I used a hydrocode specially designed to handle the extremely large gradients between the winds to second order accuracy. The outer shock shapes correspond very well to my analytic predictions. This shock may form cusps which compress the gas to form two rings on opposite sides of the equatorial plane.

\section{The Reverse Shock}

The central source becomes surrounded by a standoff shock in the fast wind. The oblateness of the slow wind causes this shock to become prolate or barrel-shaped. This causes shock focusing of the fast flow.

\section{The Contact Discontinuity}

A cool tongue-like deformation extends from the contact discontinuity, forming a chimney along the symmetry axis. Turbulent hot gas streams back from the head of the outflow. These two effects exert a confining pressure, leading to the formation of extremely well collimated jets in the fast gas.

\section{Some Observable Consequences}

The inner shock and the contact discontinuity show a very complex interplay of hydrodynamic effects. This produces a fascinating richness of internal motions and density distributions. Some of these can be readily identified with known PN features such as "helix" rings. Others are presented as predictions.

Balick, B., Preston, H.L., Icke, V.: 1987 Astron. J. 94, 1641

Icke, V.: 1987 Astron. Astrophys. 202, 177

Icke, V.: 1991 Astron. Astrophys. 251, 369

Icke, V., Balick, B., Frank, A.: 1991 Astron. Astrophys. 253, 224

Icke, V., Mellema, G., Balick, B., Eulderink, F., \& Frank, A.: 1992 Nature 355, 524.

Icke, V., Preston, H.L., Balick, B.: 1989 Astron. J. 97, 462

Mellema, G., Eulderink, F., Icke, V.: 1992 Astron. Astrophys. 252, 718 